\title{
BMJ Open Evaluating the safety and technical effectiveness of a newly developed intravascular 'flow isolator' stent for the treatment of intracranial aneurysms: study protocol for a first-in-human single-arm multiple-site clinical trial in Japan
}

\author{
Haruko Yamamoto, ${ }^{1}$ Toshimitsu Hamasaki, ${ }^{2}$ Kaori Onda, ${ }^{2}$ Yasuhide Nakayama, \\ Akira Ishii, ${ }^{3}$ Hidenori Oishi, ${ }^{4}$ Nobuyuki Sakai, ${ }^{5}$ Tetsu Satow ${ }^{6}$
}

To cite: Yamamoto $\mathrm{H}$, Hamasaki T, Onda K, et al. Evaluating the safety and technical effectiveness of a newly developed intravascular 'flow isolator' stent for the treatment of intracranial aneurysms: study protocol for a first-in-human singlearm multiple-site clinical trial in Japan. BMJ Open 2019;9:e020966. doi:10.1136/ bmjopen-2017-020966

- Prepublication history for this paper is available online. To view these files, please visit the journal online (http://dx.doi org/10.1136/bmjopen-2017020966).

Received 4 December 2017 Revised 12 February 2019 Accepted 6 March 2019

Check for updates

(C) Author(s) (or their employer(s)) 2019. Re-use permitted under CC BY-NC. No commercial re-use. See rights and permissions. Published by BMJ.

For numbered affiliations see end of article.

Correspondence to Dr Haruko Yamamoto; harukoya@ncvc.go.jp

\begin{abstract}
Introduction Wide-neck or large intracranial aneurysms are difficult to cure by conventional surgical or endovascular procedures. A flow diverter (FD) is an implantable, stent-like, fine-mesh medical device for the treatment of intracranial aneurysms. Although endovascular treatment with FDs is becoming a routine first-line option, a systematic review noted the heterogeneity and publication biases of the clinical studies for FDs. We have developed a new honeycomb microporous covered stent (NCVC-CS1) as a 'flow isolator' for the endovascular treatment of intracranial aneurysms.
\end{abstract}

Methods and analysis We planned the NCVC-CS1_UAN as a first-in-human study to evaluate the safety and technical effectiveness of the NCVC-CS1, a newly developed honeycomb microporous covered stent, for the treatment of intracranial aneurysms that are difficult to cure by conventional surgical or endovascular procedures. The study is a multicentre, open-label, uncontrolled, exploratory, medical device, investigator-initiated clinical study. The primary safety endpoint of this study is any stroke or death related to the procedure within 180 days, while for efficacy, the endpoint is complete obliteration of the target aneurysm and patency of the target vessel (less than $50 \%$ stenosis) confirmed by angiography at 180 days after the procedure.

Ethics and dissemination Full ethics approval of institutional review boards was obtained at all participating sites. A clinical trial notification as a new medical device was accepted by the Japanese regulatory agency before it started. The study should be followed by a pivotal study to obtain satisfactory data for an application for marketing approval. The main results of this study will be submitted for publication in a peer-reviewed journal. The planned subject number for this study is 12 .

Trial registration number NCT02907229.
Strengths and limitations of this study

NCVC-CS1_UAN is a first-in-human study to evaluate the safety and technical effectiveness of NCVCCS1, a newly developed honeycomb microporous covered stent, for the treatment of intracranial aneurysms that are difficult to cure by conventional surgical or endovascular procedures.

- A major strength of this study is that it evaluates a novel 'flow isolator' based on completely new technologies.

- The main limitations of this study are its open study design and the lack of control arms.

\section{INTRODUCTION}

Wide-neck or large intracranial aneurysms are difficult to cure by conventional surgical or endovascular procedures. ${ }^{1}$ A flow diverter (FD) is an implantable, stent-like, fine-mesh medical device for the treatment of intracranial aneurysms (figure 1A-C). Endovascular treatment with FDs is becoming a routine first-line option. Although some clinical studies have reported favourable results, ${ }^{2}{ }^{3}$ a systematic review noted the heterogeneity and publication biases of the clinical studies of FDs. ${ }^{4}$ Recently, a randomised trial, which was halted due to safety concerns, failed to show improvement in the expected composite primary efficacy outcome of FDs (complete or near-complete occlusion of the aneurysm combined with an independent functional outcome) compared with standard treatment, such as coil embolisation or parent artery occlusion. ${ }^{5}$ A meta-analysis of treatment outcomes of posterior circulation 


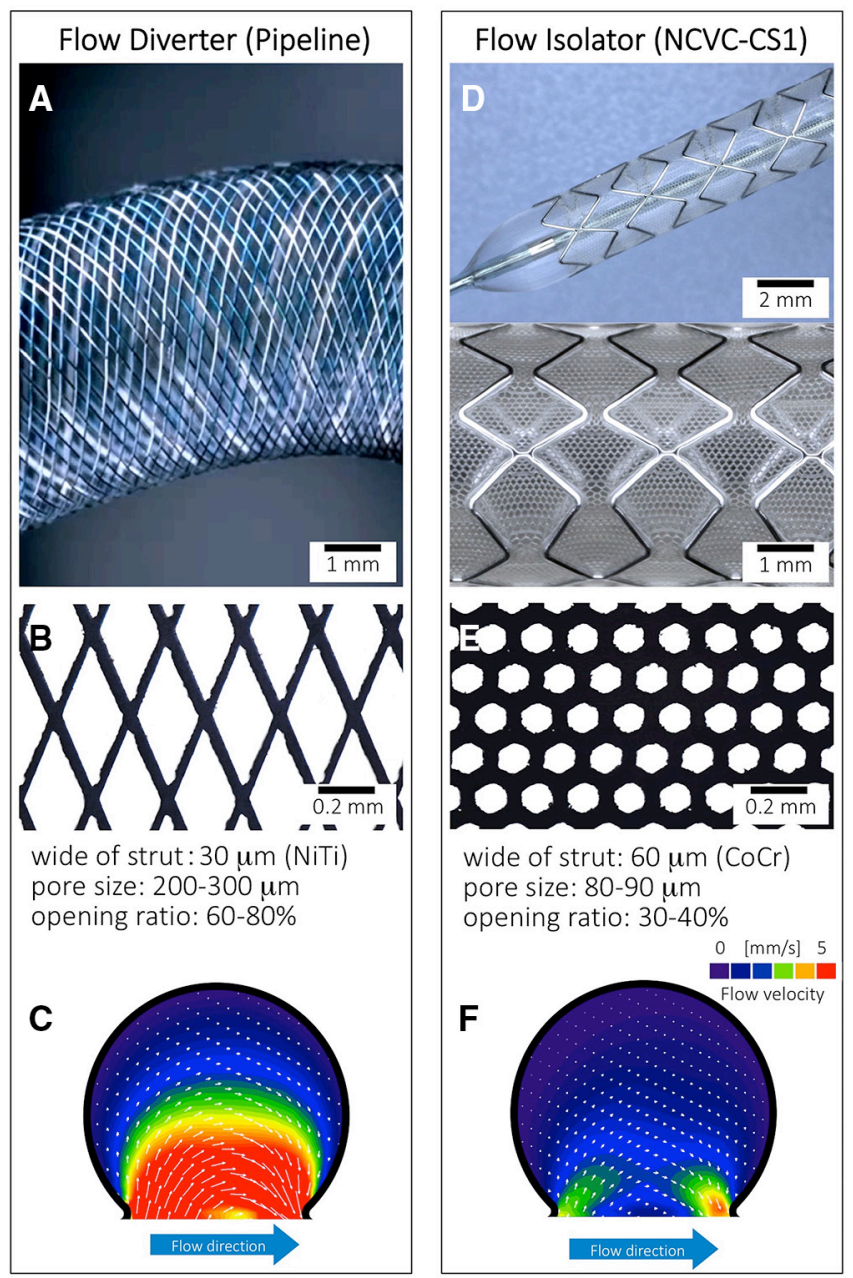

Figure 1 Comparison of specifications and in vitro performance between flow diverter $(A-C)$ and flow isolator (D-F). Macroscopic photos $(A, B)$, mesh patterns $(B, E)$ and flow velocity mappings in an aneurysm after placing a flow diverter or a flow isolator in an in vitro circuit simulating human intracranial flow (C,F). NiTi: nitinol, CoCr: cobalt chromium

non-saccular aneurysms by FDs reported a long-term occlusion rate of $52 \%$ and an overall mortality of $21 \%{ }^{6}$ Thus, a safer and more effective implantable medical device is needed.

We have developed a covered stent (NCVC-CS1) with innumerable precise micropores (pore size $80-90 \mathrm{~m}$, opening ratio $30 \%-40 \%$ ) for the endovascular treatment of intracranial aneurysms (figure 1D-F). The NCVC-CS1, a balloon-expandable cobalt chromium stent, has some structural benefits: the stents have flat luminal surfaces with a cover film, and their struts are completely impregnated in the cover film, which is extremely thin, less than $20 \mu \mathrm{m}$ thick. These advantages give high flexibility and robust properties to the NCVC-CS1.

The NCVC-CS1 showed excellent performance in our preclinical testing for the treatment of cerebral aneurysms with three important advantages. ${ }^{78}$ The first was rapid isolation of blood flow inside the aneurysms. A large portion of the aneurysm cavities disappeared immediately after stenting due to shielding of the aneurysm neck by the stents' cover film. In addition, at the 1-month follow-up, all aneurysms had almost completely disappeared. The aneurysm cavities were packed with hard thrombus, and the luminal surface of the stents was covered with a thin tissue layer including endothelial cells.

The second advantage was rapid neointimal formation with complete endothelialisation at all stented segments, which was due to the microporous polyurethane functioning as a scaffold for vascular cell migration.

The last advantage was perfect patency of the perforating arteries after stenting. In a perforating artery rabbit model, all lumbar arteries remained patent for over 1 year, even after placement of a second, overlapping NCVC-CS1. At 2 months after stenting, the luminal surface was covered with complete thin neointimal formation. In addition, even in the prototype model of the NCVC-CS1, all branches were patent in a rabbit subclavian artery model. ${ }^{9}$ Therefore, it is highly likely that the NCVC-CS1 could be appropriate for the treatment of vertebral targets incorporating the posterior inferior cerebellar artery and the basilar artery.

FDs cannot fully demonstrate the three above-mentioned advantages. Therefore, the NCVC-CS1 is expected to have a stronger therapeutic effect than conventional devices. Because the NCVC-CS1 is considered a new technology going beyond FDs, it should be called a 'flow isolator', which may be a different genre of intravascular stent than FDs.

NCVC-CS1_UAN is designed as a first-in-human study to evaluate the safety and technical effectiveness of the NCVC-CS1 for the treatment of intracranial aneurysms that are difficult to cure by conventional surgical or endovascular procedures.

\section{METHODS AND ANALYSIS \\ Study design}

NCVC-CS1_UAN is a multicentre, open-label, uncontrolled, exploratory, medical device, investigator-initiated clinical study.

\section{Study period}

The planned study period has obtained ethics approval and has been approved by the Pharmaceuticals and Medical Devices Agency (PMDA), the Japanese regulatory agency, in December 2018. Since the PMDA requires long-term follow-up, the study participants will be followed up for 5 years after stent deployment according to a separate protocol.

\section{Sample size}

The planned subject number in this study is 12 . The sample size was calculated based on the primary efficacy endpoint. A sample size of 10 would provide a two-sided $95 \%$ exact (Clopper-Pearson type) CI with a width equal to $44.2 \%$ (55.5\% to $99.7 \%$ ), assuming $90 \%$ complete obliteration of the target aneurysm and patency of the target vessel (PASS V.11; NCSS, Kaysville, UT, USA). A total of 12 patients will be recruited into the study, accounting for withdrawals from the study. 
Box 1 Inclusion and exclusion criteria of the study

\section{Inclusion criteria:}

(Provisional registration)

1. Age between 20 and 75 years.

2. Target lesion is an unruptured aneurysm regardless of prior treatment diagnosed by angiography, CT angiography, or magnetic resonance angiography within 180 days.

a. Target aneurysm located in the petrous/cavernous portion of the internal carotid artery, intradural vertebral artery except for its union or basilar artery below the orifice of the superior cerebellar artery.

b. The target aneurysm is considered difficult to treat by conventional surgical/endovascular procedures.

c. Target aneurysm has a sac diameter exceeding $7 \mathrm{~mm}$.

d. Target aneurysm has a neck diameter over $4 \mathrm{~mm}$ or with a dome/neck ratio less than 2 .

3. Modified Rankin scale score of 3 or less.

4. Agreement for participating in the study and informed consent signed by the patient.

(Final registration)

1. Target lesion must be reconfirmed by angiography or interventional procedure and is compatible with the following conditions:

a. Target aneurysm located in the petrous/cavernous portion of the internal carotid artery, intradural vertebral artery except for its union or basilar artery below the orifice of the superior cerebellar artery.

b. The target aneurysm is considered difficult to treat by conventional surgical/endovascular procedures.

c. Target aneurysm has a sac diameter exceeding $7 \mathrm{~mm}$.

d. Target aneurysm has a neck diameter over $4 \mathrm{~mm}$ or a dome/neck ratio less than 2.

e. The parent artery of the target aneurysm is accessible by the investigational devices.

f. The diameter of the parent artery is between 3.5 and $5.0 \mathrm{~mm}$.

\section{Exclusion criteria:}

(Provisional registration)

1. Any invasive surgical procedure within 30 days before registration.

2. Prior stent implantation at the site of the target aneurysm.

3. Presence of intracranial tumour, arteriovenous malformation for which any intervention is considered necessary, or coexistence of an unruptured intracranial aneurysm whose sac diameter is over $5 \mathrm{~mm}$ within the target vessel.

4. Coexistence of other unruptured intracranial aneurysms for which intervention is planned.

5. Target vessel not suitable for delivery and placement of a covered stent because of the anatomical configuration (ie, severe bend or tortuosity).

6. Stenosis of more than $50 \%$ in the target vessel or its proximity, including the extracranial region.

7. Any intracranial haemorrhage within 42 days before registration.

8. Comorbid conditions that may limit survival to less than 6 months, or difficulty in observation of the patient for 6 months.

9. Renal dysfunction with serum creatinine of more than $2.0 \mathrm{mg} / \mathrm{dL}$.

10. Contraindications to antiplatelet or anticoagulant treatment because of allergy or haemorrhagic diathesis.

11. Platelet count less than $100000 / \mathrm{mm}^{3}$ or known platelet dysfunction.

12. Active bacterial infection.

13. Any cardiac, haematological, intracranial or vessel disease considered to have a high risk of neurological events (severe cardiac failure, atrial fibrillation, arteritis and known carotid artery stenosis).

14. History of severe allergy to contrast agent.

15. Known allergy to cobalt-chromium alloy and polyurethane, clopidogrel, heparin, nitinol or local or general anaesthesia.

16. Subjects who have enrolled or planned to participate in another clinical trial or intervention study.

17. Pregnancy.

18. Patients judged unsuitable by the physician attending this trial.

(Final registration)

1. History of any invasive surgical procedure after provisional registration.

2. Any new neurological signs within 24 hours prior to the procedure.

\section{Endpoints}

The primary endpoints of this study are (1) safety endpoint, any stroke or death related to the procedure within 180 days; and (2) efficacy endpoint, complete obliteration of the target aneurysm and patency of the target vessel (less than 50\% stenosis) confirmed by angiography at 180 days after the procedure.

The secondary endpoints of this study are (1) technical success (stent placement in the target lesion covering the aneurysmal neck without occlusion of the target vessel), (2) any death within 180 days after the procedure, (3) any death due to neurological causes within 180 days after the procedure, (4) any adverse event or adverse device effect and (5) any neurological deficits.

\section{Patient enrolment and study schedule}

Complete inclusion and exclusion criteria are shown in box 1. Candidate patients who meet the inclusion and exclusion criteria for provisional registration are provisionally registered after they give informed consent. Registered patients receive dual antiplatelet therapy from at least 4 days before the study treatment. Within 


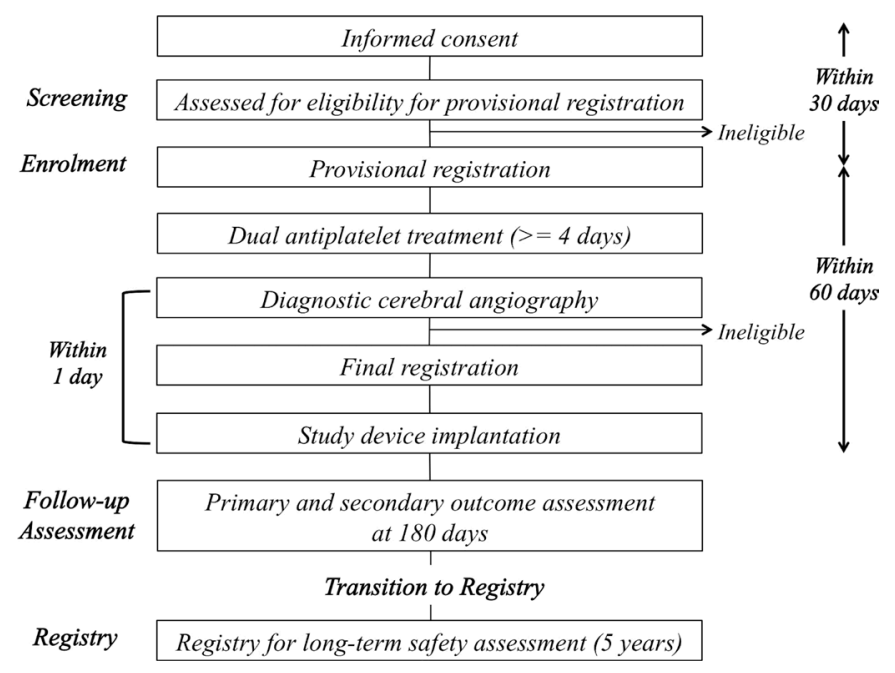

Figure 2 Summary of the study design.

60 days from provisional registration, patients undergo study device implantation after diagnostic cerebral angiography confirms that they meet the criteria for final registration. Because this is a first-in-human study, for the first three patients, enrolment is held after a patient is registered until the independent safety board confirms the 30-day safety for the patient. A summary of the study design is shown in figure 2 . The schedule of evaluation is presented in table 1 .

\section{Centres and investigators}

To join the trial, centres were required to apply for open recruitment by the Japanese Medical Association (JMA).
Participating centres must have sufficient experience and organisation to execute clinical trials. The interventional physician belonging to each centre must be a senior trainer certified by the Japanese Society for Neuroendovascular Therapy, have performed at least 300 coiling procedures for intracranial aneurysms and at least 50 stenting procedures in the intracranial vessels, and have been involved in clinical trials of preapproval neuroendovascular devices. Finally, the JMA selected the participating centres from among the centres that applied and fulfilled all requirements.

\section{Independent safety evaluation board}

The independent safety evaluation board is composed of three individuals not involved in study conduct who have expertise in multiple disciplines, including a neurosurgeon, a neurointerventionist and a neurologist. All serious adverse events are reported to and discussed by the independent safety board, and the 30-day safety of the first three patients is evaluated.

\section{Independent radiological image evaluation}

All cerebral angiography images are evaluated by an independent experienced neurointerventionist.

\section{Data management, monitoring and auditing}

The system for electronic data capture and data management is validated to meet the regulatory requirements. On-site monitoring, including source document verification and audit, is planned.

\begin{tabular}{|c|c|c|c|c|c|c|}
\hline & Entry & Treatment & 3 days & 30 days & 90 days & 180 days \\
\hline Inclusion/exclusion criteria & $\mathrm{x}$ & & & & & \\
\hline Signed consent form & $x$ & & & & & \\
\hline Provisional enrolment & $x$ & & & & & \\
\hline Medical/treatment history ${ }^{\star}$ & $x$ & & & & & \\
\hline Neurological assessment & $x$ & & $x$ & $x$ & $x$ & $x$ \\
\hline mRS score & $x$ & & & $x$ & $x$ & $x$ \\
\hline Laboratory tests $\dagger$ & $x$ & & & & & \\
\hline CTA/MRA $\ddagger$ & $x$ & & & & $x$ & \\
\hline Medication§ & $x$ & $x$ & $x$ & $x$ & $x$ & $x$ \\
\hline Cerebral angiography & & $\mathrm{x}$ & & & & $x$ \\
\hline Final enrolment & & $x$ & & & & \\
\hline Endovascular procedure ${ }^{\star \star}$ & & $x$ & & & & \\
\hline Adverse events & & $\mathrm{x}$ & $x$ & $x$ & $x$ & $\mathrm{x}$ \\
\hline
\end{tabular}

*History of cerebrovascular diseases, neurosurgical treatments, cerebral endovascular treatments, hypertension, dyslipidemia, diabetes mellitus and so on.

†Haematology, biochemistry and coagulation.

łLocation, size, form, maximum diameter, neck diameter and parent artery diameter of the target aneurysm.

§Antiplatelets, anticoagulants and medications for complications.

ILocation, size, form, maximum diameter, neck width and parent artery diameter of the target aneurysm.

${ }^{\star *}$ Site of puncture, anaesthesia and medical devices used in the procedure.

CTA, CT angiography, MRA, magnetic resonance angiography; mRS, modified Rankin scale. 


\section{Statistical analysis}

Analyses will be done in all patients recruited into the study as a primary analysis population. Patient demographic data and safety and efficacy endpoints will be analysed descriptively, with continuous data expressed as means with SD or medians with range (minimum and maximum), and categorical data will be expressed as numbers and percentages.

The primary safety and efficacy endpoints will be summarised as numbers and percentages with their two-sided $95 \%$ exact CI, using the Clopper-Pearson method. In addition, the Kaplan-Meier method will be used to estimate the event-free survivals of the safety and efficacy endpoints. For other endpoints, data will be summarised using appropriate statistics. All analyses will be performed according to a prespecified statistical analysis plan using SAS V.9.3 or later. The statistical analysis plan will be prepared separately and finalised before database locking.

\section{Patient and public involvement}

Patients or public were not involved in the design or conduct of the study, and no attempt was made to assess the burden of the intervention by patients themselves.

\section{ETHICS AND DISSEMINATION}

A clinical trial notification as a new medical device, much like a US Investigational Device Exemption application, was accepted by PMDA before it started. The study was registered in Clinicaltrials.gov and started in May 2016. After the first patient was enrolled on 12 October 2016, the new stents were implanted in eight patients by the end of November 2017. The study should be followed up by a pivotal study to obtain satisfactory data for an application for marketing approval. The long-term safety information will be obtained in a separate protocol, which is an observational one. The main results of this study and the longterm safety observational study will also be submitted for publication in a peer-reviewed journal.

\footnotetext{
Author affiliations

${ }^{1}$ Center for Advancing Clinical and Translational Sciences, National Cerebral and Cardiovascular Center, Suita, Osaka, Japan

${ }^{2}$ Department of Data Science, National Cerebral and Cardiovascular Center, Suita, Osaka, Japan

${ }^{3}$ Department of Neurosurgery, Kyoto University Graduate School of Medicine, Kyoto, Kyoto, Japan

${ }^{4}$ Department of Neurosurgery, Juntendo University Graduate School of Medicine, Bunkyo-ku, Tokyo, Japan

${ }^{5}$ Department of Neurosurgery, Kobe City Medical Center General Hospital, Kobe, Hyogo, Japan
}

${ }^{6}$ Department of Neurosurgery, National Cerebral and Cardiovascular Center, Suita, Osaka, Japan

Acknowledgements The authors would like to thank Dr Naoya Kuwayama, Dr Koji lihara and Dr Kazuo Kitagawa for their contributions as the members of the Independent Safety Evaluation Committee, and Dr Shinichi Yoshimura for his contribution as the member of the Independent Image Evaluation Committee in this trial.

Contributors YN, Al, HO, NS and TS: conception of the study. HY: writing of the manuscript. HY and KO: coordination of the study. TH: statistical analysis and providing the biostatistical study design. HY, NS and TS: members of the Steering Committee. TS: the grant holder.

Funding This research is supported by the Project Promoting Clinical Trials for Development of New Drugs and Medical Devices (Japan Medical Association) from the Japan Agency for Medical Research and Development. The study devices are produced and provided by Goodman Corporation without charge.

Competing interests None declared.

Patient consent for publication Not required.

Ethics approval Full ethics approval of the institutional review boards (IRBs) was obtained at all participating sites (National Cerebral and Cardiovascular Center IRB, Kyoto University Hospital IRB, Juntendo University Hospital IRB and Kobe City Medical Cent er General Hospital IRB).

Provenance and peer review Not commissioned; externally peer reviewed.

Open access This is an open access article distributed in accordance with the Creative Commons Attribution Non Commercial (CC BY-NC 4.0) license, which permits others to distribute, remix, adapt, build upon this work non-commercially, and license their derivative works on different terms, provided the original work is properly cited, appropriate credit is given, any changes made indicated, and the use is non-commercial. See: http://creativecommons.org/licenses/by-nc/4.0/.

\section{REFERENCES}

1. Zhao B, Yin R, Lanzino G, et al. Endovascular coiling of wide-neck and wide-neck bifurcation aneurysms: a systematic review and metaanalysis. AJNR Am J Neuroradiol 2016;37:1700-5.

2. Berge J, Biondi A, Machi P, et al. Flow-diverter silk stent for the treatment of intracranial aneurysms: 1-year follow-up in a multicenter study. AJNR Am J Neuroradiol 2012;33:1150-5.

3. Saatci I, Yavuz K, Ozer C, et al. Treatment of intracranial aneurysms using the pipeline flow-diverter embolization device: a single-center experience with long-term follow-up results. AJNR Am J Neuroradiol 2012;33:1436-46.

4. Arrese I, Sarabia R, Pintado R, et al. Flow-diverter devices for intracranial aneurysms: systematic review and meta-analysis. Neurosurgery 2013;73:193-9.

5. Raymond J, Gentric JC, Darsaut TE, et al. Flow diversion in the treatment of aneurysms: a randomized care trial and registry. $J$ Neurosurg 2017;127:454-62.

6. Kiyofuji S, Graffeo CS, Perry A, et al. Meta-analysis of treatment outcomes of posterior circulation non-saccular aneurysms by flow diverters. J Neurointerv Surg 2018;10:493-9.

7. Nakayama Y, Satow T, Funayama M, et al. Construction of 3 animal experimental models in the development of honeycomb microporous covered stents for the treatment of large wide-necked cerebral aneurysms. J Artif Organs 2016;19:179-87.

8. Nakayama Y, Satow T, Funayama M, et al. Excellent preclinical results of honeycomb microporous covered stents for the treatment of large and wide-necked cerebral aneurysms. Circulation 2015;132:A11722.

9. Nishi S, Nakayama Y, Ishibashi-Ueda H, et al. Treatment of rabbit carotid aneurysms by hybrid stents (microporous thin polyurethanecovered stents): preservation of side-branches. J Biomater Appl 2014;28:1097-104. 